Chirurg 2019 90 (Suppl 2):S30 https://doi.org/10.1007/s00104-019-0823-6 Online publiziert: 13. Februar 2019

(c) Springer Medizin Verlag GmbH, ein Teil von Springer Nature 2019

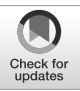

W. Schröder · C. Bruns

Klinik für Allgemein-, Viszeral- und Tumorchirurgie, Universitätsklinik Köln, Köln, Deutschland

\title{
Aktuelle Trends in der onkologischen Ösophaguschirurgie
}

\section{Eine weltweite Umfrage}

Befragten arbeiteten an einer Universitätsklinik. $72 \%$ der Teilnehmer wurden als „high volume“-Chirurgen (>21 Ösophagektomien/Jahr) klassifiziert.

Haverkamp L, Sessing MFJ, Ruurda JP, Bonne J, Hillgersberg R (2016) Worldwide trends in surgical technique in the treatment of esophageal and gastroesophageal junction cancer. Dis Esophagus. https://doi.org/10. 1111/dote.12480 [Epub ahead of print]

Hintergrund und Fragestellung. Die operativen Verfahren in der onkologischen Ösophaguschirurgie befinden sich durch die Einführung der minimalinvasiven Techniken im Umbruch, ohne dass sich bislang ein Standard etablieren konnte. Im Jahr 2007 wurde von der holländischen Arbeitsgruppe aus Utrecht eine weltweite Umfrage durchgeführt, um die unterschiedlichen chirurgischen Techniken zusammenfassend darzustellen [1]. Das Ziel der zweiten jetzt publizierten Erhebung war, Veränderungen in der Chirurgie des Ösophaguskarzinoms und Karzinoms des gastroösophagealen Übergangs im Vergleich zur ersten Umfrage von 2007 aufzuzeigen.

Methoden. Die Teilnehmer der Umfrage wurden über die Netzwerke von drei internationalen Fachgesellschaften (ISDE, OESO und IGCA) kontaktiert und gebeten, einen webbasierten Fragebogen auszufüllen. Bei einer Rücklaufquote von $42 \%$ beteiligten sich an dieser Umfrage insgesamt 478 Chirurgen aus 49 Ländern und von 6 Kontinenten. $65 \%$ der

Dieser Beitrag wurde erstpubliziert in Der Chirurg (2017) 88:74-74. https://doi.org/10. 1007/s00104-016-0358-z
Ergebnisse. In der aktuellen Umfrage wurde das Ösophaguskarzinom in $43 \%$ über einen transthorakalen minimalinvasiven Zugang operiert, in $38 \%$ präferierten die Chirurgen einen offen transthorakalen Zugang, der transhiatale $\mathrm{Zu}$ gang wurde von $19 \%$ der Befragten gewählt. Im Vergleich zu 2007 verdreifachte sich damit die Rate der minimalinvasiv operierten Patienten. Insbesondere bei den „high volume“-Chirurgen wurde der minimalinvasive Zugang in über $50 \%$ der Patienten praktiziert. In über $95 \%$ erfolgte die Rekonstruktion mittels Magenhochzug. Im Beobachtungszeitraum von 7 Jahren gab es eine Verschiebung von der zervikalen zur intrathorakalen Rekonstruktion, die beim offenen Vorgehen in 68 \% (2007: $44 \%)$ und beim minimalinvasiven in $46 \%$ der Patienten (2007: $15 \%$ ) praktiziert wurde. $77 \%$ der intrathorakalen Anastomosen wurden mittels Stapler angelegt. In Europa und Nordamerika wurden fast ausschließlich 2-Feld-Lymphadenektomien durchgeführt, während in Asien in über $40 \%$ der Patienten die Lymphadenektomie um das zervikale Kompartiment erweitert wurde.

Fazit. Trotz ihrer Komplexität gewinnt die minimalinvasive transthorakale Ösophagektomie bei den onkologischen Erkrankungen im klinischen Alltag zunehmend an Bedeutung und kommt mittler- weile in fast der Hälfte aller operierten Patienten zur Anwendung. Die vorliegende Umfrage legt nahe, dass die Selektion der Patienten für die minimalinvasiven Techniken insbesondere durch die technischen Fähigkeiten des einzelnen Chirurgen und damit die individuelle Lernkurve bestimmt wird. In Abhängigkeit von dieser Lernkurve ist zu erwarten, dass die Rate der minimalinvasiven Verfahren auch weiter zunehmen wird. Kritisch anzumerken ist, dass in dieser Umfrage nicht zwischen den total minimalinvasiven und den Hybridverfahren (Laparoskopie/Thorakotomie) unterschieden wird. Diese Verfahren können vergleichend hinsichtlich ihres frühpostoperativen Outcomes und der Langzeitergebnisse zum gegenwärtigen Zeitpunkt noch nicht abschließend bewertet werden. Die Zunahme von „high volume“Chirurgen ist Ausdruck der zunehmenden Zentralisierung von Patienten mit Ösophaguskarzinom.

\section{Korrespondenzadresse}

Prof. Dr. W. Schröder, FACS

Klinik für Allgemein-, Viszeral- und Tumorchirurgie, Universitätsklinik Köln Kerpener Str. 62, 50937 Köln, Deutschland wolfgang.schroeder@uni-koeln.de

Interessenkonflikt. W. Schröder und C. Bruns geben an, dass kein Interessenkonflikt besteht.

\section{Literatur}

1. Boone J, Livestro DP, Elias SG, Borel Rinkes IH, von Hillegersberg R (2009) International survey on esophageal cancer: part I surgical techniques. Dis Esophagus 22:195-202 